BULLETIN OF THE

AMERICAN MATHEMATICAL SOCIETY

Volume 78, Number 4, July 1972

\title{
LOCALIZATION AND COMPLETION
}

\author{
BY J. LAMBEK
}

Communicated by Alex Rosenberg, December 27, 1971

Let $I$ be an injective right $R$-module and $E$ its ring of endomorphisms. The functor

$$
\operatorname{Hom}_{R}(-, I): \operatorname{Mod} R \rightarrow(E \mathrm{Mod})^{\text {op }}
$$

has a right adjoint $\operatorname{Hom}_{E}(-, I)$, giving rise to a triple (standard construction) $(S, \eta, \mu)$, where

$$
S=\operatorname{Hom}_{E}\left(\operatorname{Hom}_{R}(-, I), I\right) .
$$

Let $Q \rightarrow S$ be the equalizer of the pair of maps $\eta S, S \eta: S \rightarrow S^{2}$, then $Q$ is the bext co-approximation of $S$ by an idempotent triple [2].

$Q(M)$ is called the localization or module of quotients of $M$ at $I$, and $Q(R)$ is a ring, the ring of quotients. $Q(M)$ may also be described as the divisible hull of $M$ made torsionfree, in the torsion theory obtained from $I$ (see e.g. [4]). In this torsion theory one calls $M$ torsion if $\operatorname{Hom}_{R}(M, I)=0$, torsionfree if $M$ is isomorphic to a submodule of a power of $I$, divisible if $I(M) / M$ is torsionfree, where $I(M)$ is the injective hull of $M$.

The endofunctor $Q$ of $\operatorname{Mod} R$ is always left exact. It is isomorphic to the identity functor if and only if it may be obtained from

$$
I=\operatorname{Hom}_{R}(F, Q / Z),
$$

where $F$ is a free left $R$-module. $Q$ is isomorphic to $(-) \otimes_{R} Q(R)$ if and only if it may be obtained from $I=\operatorname{Hom}_{R}(F, Q / Z)$, where $R \rightarrow F$ is an epimorphism of rings and $F$ is a flat left $R$-module [4], [7], [10]. $Q$ is exact if and only if $I(M) / M$ is divisible for all torsionfree divisible modules $M$ (compare with [3]), and then $Q(M) \cong M \otimes_{R} Q(R)$ for every finitely presented module $M$. We also note that every divisible module is injective if and only if $I$ has zero singular submodule.

The $I$-adic topology on $M$ is defined by taking as fundamental open neighborhoods of zero all kernels of homomorphisms $M \rightarrow I^{n}$, where $n$ is finite. This topology and its relation to the usual $P$-adic topology has been discussed in [5].

The torsion submodule of $M$ is open in the $I$-adic topology if and only if $\operatorname{Hom}_{R}(M, I)$ is a finitely generated $E$-module, or, equivalently, $\operatorname{Hom}_{R}\left(M, I^{n}\right)$ is a principal $\operatorname{End}_{R}\left(I^{n}\right)$-module for some finite $n$. These 
conditions imply that $Q(M)=S(M)$. Since $S(R)$ is the bicommutator of $I$, one obtains known results by Morita and the author as special cases.

While $Q(M)$ is a closed submodule of $S(M)$ in the $I$-adic topology, one obtains a density theorem when $S(M)$ is endowed with the finite topology: the topology induced by the product topology of $I^{\mathrm{Hom}_{R}(M, I)}$ when $I$ is discrete.

THEOREM. When $Q$ is exact, $S(M)$ with the finite topology is the completion of $Q(M)$ with the I-adic topology.

For $M=R$, this specializes to a known result [5] about the bicommutator of $I$.

The proof of this theorem makes use of certain algebras and homomorphisms of the triple $(S, \eta, \mu)$ in the sense of [1]. When $Q$ is exact, the algebras of this triple may be characterized as torsionfree divisible modules equipped with a limit operation $\lambda$ which assigns a limit to each $I$-adic Cauchy net. One requires that $\lambda$ is $R$-linear, that it sends every convergent net to its usual limit, and that the limit of a product net may be computed as an interated limit.

\section{REFERENCES}

0. M. F. Atiyah and I. G. Macdonald, Introduction to commutative algebra, AddisonWesley, Reading, Mass., 1969. MR 39 \# 4129.

1. S. Eilenberg and J. C. Moore, Adjoint functors and triples, Illinois J. Math. 9 (1965), 381-398. MR 32 \# 2455.

2. S. Fakir, Monade idempotente associeé à une monade, C. R. Acad. Sci. Paris Sér. A-B 270 (1970), A99-A101. MR 41 \# 1828.

3. O. Goldman, Rings and modules of quotients, J. Algebra 13 (1969), 10-47. MR 39 \# 6914

4. J. Lambek, Torsion theories, additive semantics, and rings of quotients, Lecture Notes in Math., no. 177, Springer-Verlag, Berlin and New York, 1971.

5. - Bicommutators of nice injectives, J. Algebra 21 (1972), 60-73.

6. E. Matlis, Injective modules over Noetherian rings, Pacific J. Math. 8 (1958), 511-528. MR 20 \# 5800.

7. K. Morita, Flat modules, injective modules and quotient rings, Math. Z. 120 (1971), $25-40$.

8. B. J. Müller, Linear compactness and Morita duality, J. Algebra 16 (1970), 60-66. MR 41 \# 8474.

9. B. Rattray, Non-additive torsion theories (to appear).

10. B. Stenström, $A$ survey of the theory of rings of quotients, Lecture Notes in Math., no. 237, Springer-Verlag, Berlin and New York, 1971.

Department of Mathematics, McGill University, Montreal, Canada 